\title{
Assistência de enfermagem aos portadores de Hipertensão Arterial Sistêmica: uma
}

\section{revisão integrativa}

\author{
Nursing assistance for people with Systemic Arterial Hypertension: an integrative review \\ Cuidados de enfermería a pacientes con Hipertensión Arterial Sistémica: una revisión integradora
}

Recebido: 10/08/2021 | Revisado: 18/08/2021 | Aceito: 17/09/2021 | Publicado: 18/09/2021

\author{
Ana Júlia da Silva Nogueira \\ ORCID: https://orcid.org/0000-0001-9191-9685 \\ Universidade Estadual da Paraíba, Brasil \\ E-mail: anajuliangr51@gmail.com \\ Jéssica Larissa Viana Silva \\ ORCID: https://orcid.org/0000-0002-1823-3529 \\ Universidade Estadual da Paraíba, Brasil \\ E-mail: vianas.jessica@gmail.com \\ Clésia Oliveira Pachú \\ ORCID: https://orcid.org/0000-0002-7356-6297 \\ Universidade Estadual da Paraíba, Brasil \\ E-mail: clesiapachu@hotmail.com
}

\begin{abstract}
Resumo
A Hipertensão Arterial Sistêmica (HAS) representa condição clínica multifatorial caracterizada pela elevação sustentada dos níveis pressóricos $\geq 140$ e/ou $90 \mathrm{mmHg}$. O elevado número de casos e as baixas taxas de controle da patologia a configuram como fenômeno ascendente, constituindo-se num grave problema de Saúde Pública no país. Objetivou-se analisar a assistência de enfermagem aos portadores de hipertensão arterial por meio de uma revisão integrativa da literatura científica. Foi realizada uma revisão integrativa da literatura por meio de busca sistematizada de artigos em periódicos indexados nas bases de dados: LILACS e SciELO, publicados nos últimos 5 anos. A presente pesquisa foi efetivada no período de fevereiro a março de 2021. Foram utilizados 12 artigos publicados no Brasil e/ou por brasileiros. A técnica de análise dos conteúdos utilizados foi relacionada mediante os critérios de exclusão e inclusão dos objetivos do estudo. Com base na sistematização desse conhecimento construído ficou evidente que os cuidados de enfermagem ao paciente com hipertensão arterial sistêmica são prestados por meio da educação em saúde. Bem como, obtendo um diagnóstico preciso para análise de adesão ao tratamento, medicamentoso ou não medicamentoso. Ademais, torna-se importante saber que toda essa integralidade do cuidado ao paciente se dá sempre prezando pelo bem-estar sócio emocional, tendo em vista que lidar com a cronicidade da Hipertensão se torna algo complexo, porquanto o tratamento perdura por toda a vida.
\end{abstract}

Palavras-chave: Cuidados de enfermagem; Hipertensão; Assistência de enfermagem.

\begin{abstract}
Systemic arterial hypertension (SAH) represents clinical condition multifactorial characterized by in the blood pressure $\geq 140 \mathrm{and} / \mathrm{or} 90 \mathrm{mmHg}$. The high number of cases and the low control rates of the pathology configure it as an ascendant phenomenon, constituting a serious Public Health problem in the country. The objective was to analyze nursing care for people with hypertension through an integrative review of the scientific literature. An integrative literature review was carried out by systematically searching for articles in journals indexed in the LILACS and SciELO databases, published in the last 5 years. The present search was carried out in the period February to March 2021. We used 12 articles published in Brazil and/or by Brazilians.The content analysis technique used was related through the exclusion and inclusion criteria of the study objectives. Based on the systematization of this built knowledge it was evident that nursing care to the patient with systemic arterial hypertension is provided through healthiness education. As well as, obtaining an accurate diagnosis for analysis of adherence to treatment, medicated or non- medicated. Furthermore, it is important to know that all this integrality of patient care is always done with a focus on socio-emotional well-being, considering that dealing with the chronicity of hypertension becomes somewhat complex, since the treatment lasts a lifetime.
\end{abstract}

Keywords: Nursing care; Hypertension; Nursing assistance.

\section{Resumen}

La Hipertensión Arterial Sistémica (HAS) representa una condición clínica multifactorial caracterizada por la elevación sustentada en los niveles presóricos $\geq 140$ y/o $90 \mathrm{mmHg}$. El elevado número de casos y las bajas tasas de control de la patología la configuran como un fenómeno ascendente, constituyendo un grave problema de salud pública en el país. El objetivo es analizar la asistencia sanitaria a los portadores de hipertensión arterial mediante una 
revisión integradora de la literatura científica. Se realizó una revisión bibliográfica integradora a través de una búsqueda sistemática de artículos en revistas indexadas en las bases de datos LILACS y SciELO, publicados en los últimos 5 años. Esta investigación se llevó de febrero a marzo de 2021. Se utilizaron doce artículos publicados en Brasil y/o por brasileños. La técnica de análisis de contenido utilizada se relacionó a través de los criterios de exclusión e inclusión de los objetivos del estudio. A partir de la sistematización de este conocimiento construido se evidenció que el cuidado de enfermería al paciente con hipertensión arterial sistémica se realiza a través de la educación para la salud. Así como, obtener un diagnóstico preciso para analizar la adherencia al tratamiento, farmacológico o no farmacológico. Además, es importante saber que toda esta integralidad de la atención al paciente se da siempre valorando el bienestar socioemocional, considerando que el abordaje de la cronicidad de la hipertensión se vuelve algo complejo, ya que el tratamiento dura toda la vida.

Palabras clave: Cuidados de enfermeira; Hipertensión; Asistencia de enfermería.

\section{Introduçãa}

A $7^{\circ}$ Diretriz Brasileira de Hipertensão Arterial (2017) infere que a Hipertensão Arterial Sistêmica (HAS) é uma condição clínica multifatorial caracterizada pela elevação sustentada dos níveis pressóricos $\geq 140$ e/ou $90 \mathrm{mmHg}$. Frequentemente se associa a distúrbios metabólicos, alterações funcionais e/ou estruturais de órgãos-alvo, sendo agravada pela presença de outros fatores de risco (FR), como dislipidemia, obesidade abdominal e diabetes mellitus (DM).

Segundo a Sociedade Brasileira de Cardiologia (2020) os principais fatores de risco para a HAS são: idade, sexo e etnia, sobrepeso e obesidade, consumo elevado de sal e álcool, sedentarismo e fatores socioeconômicos intrinsecamente relacionados aos determinantes e condicionantes de saúde.

As Doenças Cardiovasculares (DCV) são as principais causas de mortes prematuras em todo o mundo. No Brasil, a HAS é a DCV mais frequente e segundo dados do Sistema de Informação de Mortalidade (SIM) a HAS foi responsável por cerca de 302 mil óbitos apenas no ano de 2017. O elevado número de casos e as baixas taxas de controle da patologia a configuram como um fenômeno ascendente e que constitui um grave problema de Saúde Pública no país (Ministério da Saúde, 2019).

A $7^{\circ}$ Diretriz Brasileira de Hipertensão Arterial (2016) aponta dois métodos terapêuticos que seriam o tratamento não medicamentoso, e o tratamento medicamentoso. Dessa forma, o tratamento não medicamentoso visa a orientação, objetivando a mudança do estilo de vida, seja por meio de medidas nutricionais, prática de atividades físicas, cessação do tabagismo, controle de estresse, entre outros. Já o tratamento medicamentoso orienta o paciente sobre a importância da utilização contínua do medicamento, bem como a possibilidade do aparecimento de efeitos adversos. O tratamento da HAS tem como objetivo diminuir a morbimortalidade cardiovascular.

A HAS é uma doença crônica e por esse motivo o seu tratamento perdura por toda a vida. Destarte, exige de uma equipe multiprofissional para garantir o melhor tratamento e controle para o hipertenso. O enfermeiro pode realizar consulta de enfermagem regulamentada pelo Conselho Federal da profissão por meio da Resolução no 358/2009, a qual dispõe sobre a implementação do Processo de Enfermagem em ambientes, públicos ou privados, em que ocorre o cuidado realizado por enfermeiros, permitindo o levantamento de fatores de risco e complicações da HAS. Assim, a finalidade deste artigo é mostrar a prevalência da assistência de enfermagem ao portador de HAS, analisando a forma de orientação e exibição do conhecimento, desmistificando assim o acesso às informações prestadas ao paciente.

\section{Metodologia}

Trata-se de um estudo de coleta de dados, obtido através de fontes secundárias por meio de levantamento bibliográfico para a realização de uma revisão integrativa da literatura. A revisão integrativa é a mais ampla abordagem metodológica, no que se refere às revisões, pois possibilita a inclusão de estudos experimentais e não-experimentais, 
proporcionando uma análise completa da temática estudada (Souza, Pessoa, Oliveira, Meneses, Costa, Alves \& Almeida, 2019).

Foi realizado um levantamento de artigos científicos nos bancos de dados Literatura Latino-americana e do Caribe em Ciências da Saúde (LILACS) e Scientific Eletronic Library Online (SciELO), no período de Fevereiro a Março de 2021. Para isto, as etapas a seguir foram realizadas: 1) Identificação da questão norteadora. 2) Definição dos descritores a serem utilizados na busca: Cuidados de Enfermagem; Hipertensão, intercalados pelo operador booleano “AND” e indexado nos Descritores em Ciências da Saúde (DeCS). 3) Seleção da amostragem após determinação dos critérios de inclusão, no qual considerou-se os estudos disponíveis na íntegra, em português e publicados nos últimos 5 anos. 4) após a realização de cada busca, a seleção inicial ocorreu mediante leitura prévia dos títulos e resumos dos artigos encontrados, tendo como critérios de exclusão: estudos repetitivos, e que não se relacionassem com a temática estudada. 5) caracterização e sistematização dos resultados. 6) a apresentação, interpretação e discussão dos dados.

Ao realizar as buscas, inicialmente foram encontrados 544 artigos, destes 9 na SciELO e 535 no LILACS. Após aplicar os critérios de exclusão e avaliar a elegibilidade dos artigos, foram selecionados 12 estudos que atendiam aos critérios desta pesquisa. As etapas que foram seguidas podem ser observadas no fluxograma a seguir (Figura 1).

Figura 1 - Representação esquemática da metodologia utilizada ao realizar a busca dos artigos selecionados e a amostra final, no período de Fevereiro a Março de 2021.

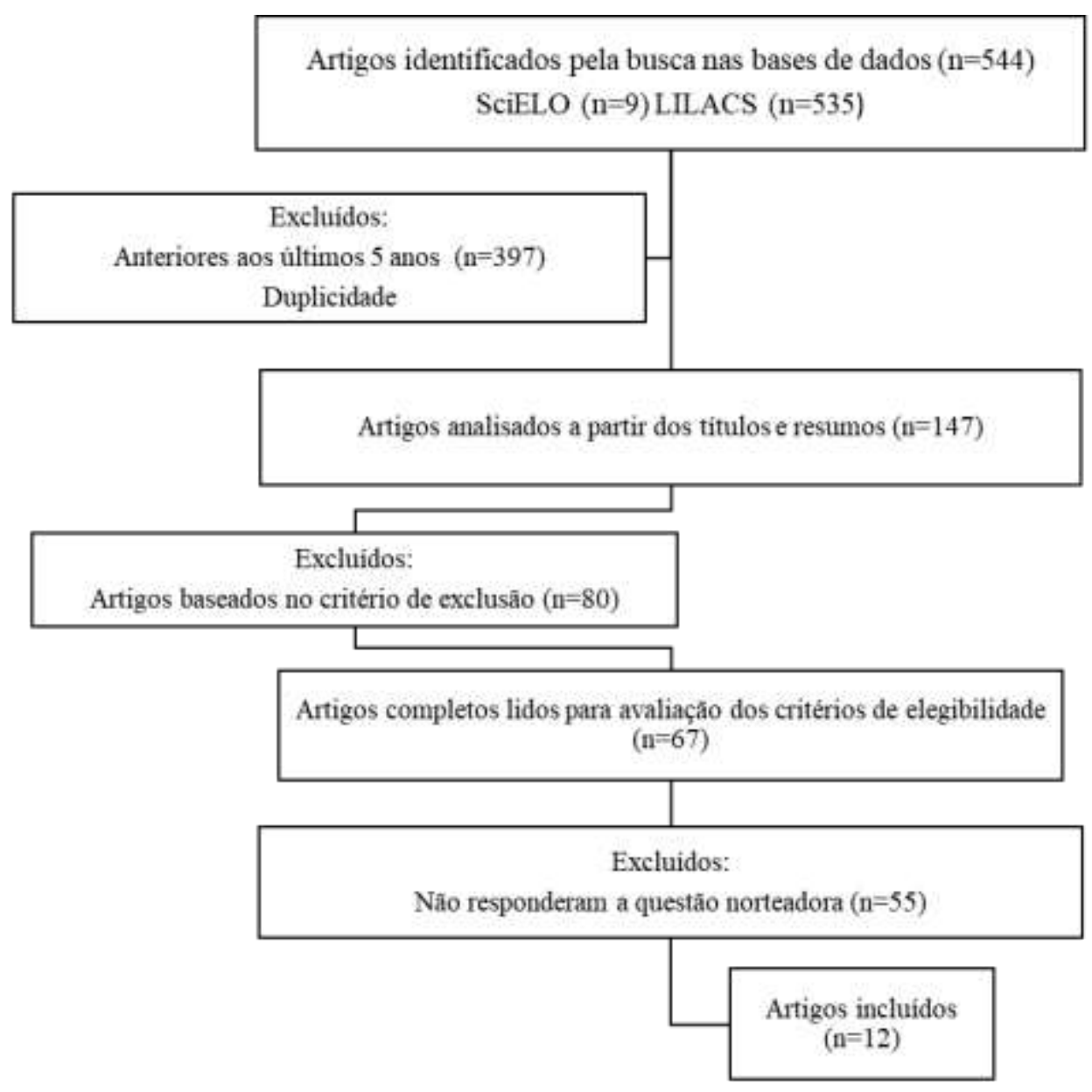

Fonte: Autores (2021). 


\section{Resultados}

Foram encontrados 544 artigos nos bancos de dados Lilacs e Scielo, após a aplicação dos critérios de inclusão e exclusão a amostra final do estudo foi composta por 12 artigos. No Quadro 1 foram listados os artigos que compuseram a amostra.

Tabela 1 - Categorização dos artigos selecionados para compor a amostragem do estudo.

Título Autor(es) $\quad$ Ano $\begin{gathered}\text { Base de } \\ \text { dados }\end{gathered} \quad \begin{gathered}\text { Delineamento } \\ \text { de estudo }\end{gathered}$ Desfecho

\begin{tabular}{|c|c|c|c|c|c|c|}
\hline A1 & $\begin{array}{l}\text { Avaliação da satisfação } \\
\text { do resultado de } \\
\text { enfermagem Bem-estar } \\
\text { Pessoal em idosos com } \\
\text { doenças crônicas }\end{array}$ & $\begin{array}{l}\text { Cavalcante, } \\
\text { Oliveira, } \\
\text { Gondim, } \\
\text { Ferreira, Nemer, } \\
\text { \& Moreira }\end{array}$ & 2020 & LILACS & $\begin{array}{l}\text { Descritivo } \\
\text { transversal, com } \\
\text { abordagem quali- } \\
\text { quantitativa }\end{array}$ & $\begin{array}{l}\text { A assistência de enfermagem e o suporte } \\
\text { necessário garantem bem estar pessoal } \\
\text { satisfatório aos idosos portadores de doenças } \\
\text { crônicas. }\end{array}$ \\
\hline
\end{tabular}

A2 O enfermeiro e a questão da adesão do paciente ao tratamento hipertensão

da
sistêmica

A3 Efetividade da consulta de enfermagem na adesão ao tratamento da hipertensão arterial sistêmica

A4 Associação entre adesão ao tratamento antihipertensivo integralidade atendimento enfermeiros

Métodos não
farmacológicos utilizados
pelo Enfermeiro na
prevenção e controle da
Hipertensão Arterial
Sistêmica

A6 Cuidados de enfermagem para pessoas com diabetes mellitus e hipertensão arterial: Mapeamento cruzado

A7 Caracterização sociodemográfica e clínica dos pacientes hipertensos não controlados atendidos em uma unidade de pronto atendimento
Salles, Sampaio,

Pereira,

Malheiros, \&

Gonçalves

Amaral-

Moreira,

Moura-Lanza \&

Nogueira-

Cortez

Sousa, Moreira,

Machado, \&

Silva

no

de

Silva, Silva,

Souza, \& Cunha

2018 LILACS

Descritivo abordagem quantitativa

Vieira,

Azevedo,

Sampaio,

Oliveira,

Moraes \& Mata
2019 LILACS Abordagem qualitativa

Abordagem quantitativa

\section{SciELO Estudo transversal}

A assistência de enfermagem qualificada e a implementação de estratégias fortalecem a adesão ao tratamento medicamentoso da Hipertensão e reduz suas complicações e custos

2019 SciELO Ensaio clínico A assistência sistematizada de enfermagem não-controlado pode beneficiar pessoas diagnosticadas com hipertensão arterial sistêmica

Há uma necessidade de realizar um cuidado de enfermagem centrado na integralidade da assistência, de forma a buscar cada vez mais a adesão dos hipertensos ao tratamento.

O tratamento não farmacológico implementado pelo Enfermeiro consiste na educação em saúde que busca maior adesão ao tratamento e modificações comportamentais para efetiva redução dos fatores de risco para a hipertensão.

Os principais cuidados de enfermagem foram as precauções cardíacas, aconselhamento nutricional, ensino sobre medicamentos prescritos e controle da nutrição.
Sousa, Pessoa, 2019 LILACS Descritivo, Oliveira,

Meneses, Costa, Alves, \&

Almeida. transversal quantitativo
É necessário a realização de pesquisas, e planejamento, execução e avaliação das medidas de educação em saúde para o controle da hipertensão arterial 
A8 Cuidados de enfermagem em crise hipertensiva: uma revisão integrativa

A9

Diagnósticos
enfermagem

portadores de hipertensão

arterial na atenção

primária
Daniel, Pedrosa, 2020 LILACS

\& Veiga

Mendes, Silva, 2018 SciELO \& Ferreira
Revisão integrativa literatura

Revisão integrativa literatura
Sousa, Moreira, 2018 LILACS Abordagem Machado, \& quantitativa ao tratamento antihipertensivo integralidade atendimento e no de enfermeiros Silva

\section{A11 Sistematização Assistência \\ Enfermagem na Atença Primária a pessoas portadoras de hipertensão arterial \\ da}

A12 Educação em saúde na atenção básica: uma análise das ações com hipertensos Rodrigues
Abordagem descritiva
Vasconcelos,

Farias,

Nascimento,

Cavalcante,

Mira \& Queiroz
É necessária a realização de estudos que da abordam a atuação do enfermeiro frente aos cuidados prestados

A identificação dos diagnósticos de da enfermagem melhora $o$ atendimento pois promove um cuidado de enfermagem planejado, facilitando sua implementação e favorecendo a avaliação do mesmo, contribuindo para redução da morbimortalidade advinda de complicações da HAS.

Verifica-se a necessidade de se realizar um cuidado de enfermagem centrado na integralidade da assistência, de forma a buscar cada vez a adesão dos pacientes hipertensos ao tratamento.

A sistematização da assistência de enfermagem ao hipertenso em alto risco cardiovascular na Atenção Primária representa uma metodologia para subsidiar as ações de promoção à saúde e prevenção dos agravos.

O desenvolvimento de ações educativas voltadas à clientela hipertensa tem contribuído na qualificação do fazer profissional e no aprimoramento dos serviços de saúde

Fonte: Autores (2021).

\section{Discussão}

A partir dos dados obtidos e apresentados (Tabela 1) foram observados que o enfermeiro é parte integrante da equipe multiprofissional e realiza a assistência de enfermagem aos portadores de HAS através da educação em saúde, avaliando as práticas, promovendo uma maior adesão ao tratamento e consequentemente reduzindo os agravos relacionados à HAS.

A educação em saúde é um dos principais dispositivos para viabilizar a promoção da saúde na Atenção Básica (AB) no Brasil e constitui-se como uma estratégia no cuidado à clientela hipertensa, atuando na prevenção e redução dos agravos decorrentes da doença (Vasconcelos, Farias, Nascimento, Cavalcante, Mira \& Queiroz., 2017). Diante disso, o artigo A11 também destaca a importância do papel da $\mathrm{AB}$ ao subsidiar as ações de promoção à saúde e prevenção dos agravos.

O artigo A5 evidenciou que a HAS é um grave problema de saúde pública no Brasil e no mundo. Sua prevalência nos indivíduos entre 60 a 69 ultrapassam os $50 \%$, podendo chegar a mais de $75 \%$ em indivíduos com idade superior a 70 anos. Evidências indicam que o aumento das Doenças Crônicas Não Transmissíveis (DCNTs), sobretudo, da HAS se dá em função do crescimento dos principais fatores de risco (Malta, Silva, Moura \& Neto, 2018). Desse modo, a intervenção direta nos fatores de risco a partir de ações de educação em saúde realizadas pelo profissional enfermeiro resultaria em redução no surgimento de novos casos da doença.

As DCNTs são um importante problema de saúde pública, e cresce em concomitância ao processo de envelhecimento da população. Os estudos A1 e A7 aqui analisados evidenciaram uma predominância significativa de idosos acometidos por doenças crônicas, principalmente a HAS. Diante disso, entender e avaliar a existência da necessidade do cuidado do profissional de enfermagem é primordial, visto que, o apoio ofertado por estes profissionais eleva o bem-estar pessoal do 
idoso, levando-o a avaliar sua vida de modo mais positivo, independente da presença de comorbidades (Cavalcante et al., 2020).

O enfermeiro é um educador em potencial, e exerce esse papel na assistência em grupos de pessoas hipertensas, no âmbito familiar e na comunidade. Além disso, é o responsável por desenvolver a consulta de enfermagem e o processo de enfermagem (P.E), identificando os principais fatores de risco e propondo mudanças individuais pautadas na singularidade de cada sujeito. Conforme foi evidenciado no artigo A5, no estudo de Chaves, Silva, Motta, Ribeiro \& Andrade (2016), também se percebeu a importância de empregar a Sistematização da Assistência de Enfermagem (SAE) e o P.E para subsidiar o enfermeiro e torná-lo capaz de prestar o cuidado de forma integral e com qualidade.

O artigo A6 potencializa também que os cuidados prescritos por enfermeiros sejam fundamentais para promoção, prevenção e reabilitação da saúde, pois a consulta de enfermagem permite o levantamento de fatores de risco e complicações da HAS. Dessa forma, é sabido que a HAS é um importante fator de risco (FR) cardiovascular e torna-se mais preocupante quando associada a outros fatores de risco (Sociedade Brasileira de Cardiologia, 2016).

$\mathrm{O}$ artigo A2 evidenciou que para a adesão ao tratamento é necessário que o paciente esteja orientado e ciente das contribuições das medidas preventivas para controle da HAS, transmitidas nas consultas e orientações de enfermagem intimamente ligadas à terapia medicamentosa e às mudanças no estilo de vida. Dessa forma, o artigo A5 mostra que estimular o paciente a ter hábitos de vida saudáveis com medidas de promoção à saúde através de atividades educativas é imprescindível. A eficácia comprovada dos hábitos saudáveis na queda de valores pressóricos, na diminuição do risco para eventos cardiovasculares e para a síndrome metabólica. Destacam-se a redução do peso, restrição do sódio, a dieta e a prática regular da atividade física como medidas comprovadas para diminuir a pressão arterial.

É importante submeter métodos mais ativos, que possibilitem o envolvimento de todos os presentes na ação e que promovam mudança, por meio de uma troca de conhecimentos, implicando em aprendizado e adoção de novos hábitos. As rodas de conversas e oficinas refletem esse novo olhar que é dado à educação em saúde, pois tem como objetivo dinamizar os momentos de grupos, nos quais a clientela hipertensa está envolvida, bem como de subsidiar o trabalho desenvolvido pelos profissionais de saúde, oferecido na atenção primária (Vasconcelos et al., 2017).

$\mathrm{O}$ artigo A3 ressalta que os usuários se sentem valorizados e cuidados quando os atendimentos são humanizados, individualizados e com ações educativas em grupo, e isso intensifica o vínculo da equipe de saúde com a comunidade, aumentando a adesão ao tratamento. O artigo A10 evidenciou que a integralidade e a interdisciplinaridade do cuidado, é remeter à realização de práticas intersubjetivas por meio do estabelecimento de relações interpessoais entre os sujeitos, pelas quais se alcança o sucesso com práticas de saúde dialógicas, principalmente quando estas se estabelecem entre enfermeiros e as pessoas que convivem com a HAS, pois nesse caso o convívio é mais intenso e estreito.

Com base nos artigos A5, A6, A11 e A12 foram analisados que os principais cuidados de enfermagem são pautados na educação em saúde e no diagnóstico de enfermagem. Dessa forma, foi encontrado na literatura um estudo de Maia, Silva, Melo, Rodrigues \& Júnior (2018) que também evidencia a educação em saúde a pacientes hipertensos como forma de aumentar a adesão ao tratamento. Sendo assim, este estudo constatou que existe o modelo tradicional de trazer informações ao paciente como forma de palestras, sendo necessário modificar, porquanto é mister estimular os protagonismos dos indivíduos motivando ao exercício de práticas mais dinâmicas, como relata o artigo A12.

A análise do estudo de Lima, Balbino, Souza \& Salgado (2017), também dinamizou assim como os artigos A3, A9 e A10 que o diagnóstico de enfermagem é de suma importância para os sujeitos portadores de hipertensão arterial sistêmica. Os diagnósticos seguem uma ordem de prioridade e ponderam o grau de comprometimento do nível do bem-estar do paciente, sendo elaborados com base no livro NANDA (Herdman \& Kamitsuru, 2018). O artigo A9 ainda ressalta que a identificação dos diagnósticos de enfermagem, melhora o atendimento para o usuário hipertenso, pois promove um cuidado planejado, 
facilitando sua implementação e favorecendo a avaliação do mesmo, contribuindo para redução da morbimortalidade advinda de complicações da HAS, provenientes da falta de adesão pelos hipertensos na Atenção Primária. Dessa forma, existe sempre a necessidade de prezar pelo melhor atendimento ao paciente, corroborando assim para uma prática integrativa de inserção no tratamento adequado, valorizando a humanização, ou seja, acolhendo o portador de HAS com uma melhor conduta.

Destarte, ficou evidente que diante de todas as análises dos artigos o cuidado de enfermagem se dá principalmente na Atenção Primária demonstrando que a sistematização do cuidado fica por meio do embasamento de práticas integrativas visando o portador de HAS como protagonista, por meio de dinamicidade no tratamento e acompanhamento da evolução constante.

\section{Considerações Finais}

Este estudo permitiu analisar e identificar os principais cuidados prestados na assistência de Enfermagem ao portador de hipertensão arterial sistêmica, no qual, a literatura evidenciou maior ênfase à educação em saúde, sobretudo, na Atenção Primária.

É sabido que o hipertenso necessita de um cuidado integral, tendo em vista a cronicidade da doença e os seus agravos, diante disso, o incentivo a adoção às práticas alimentares saudáveis é fundamental, bem como, a sensibilização acerca da importância da adesão ao tratamento para controle da HAS e prevenção de agravos.

Dessa forma, o trabalho da enfermagem com os novos métodos obteve boas perspectivas em relação ao cuidado do paciente com HAS, porquanto permitiu desmistificar os conhecimentos, garantindo assim o bem-estar social e emocional do indivíduo para lidar com a cronicidade da doença de uma forma leve.

A partir deste estudo, também foi possível observar um número ínfimo de publicações atuais que se relacionam diretamente a essa temática, o que representa uma limitação importante na construção deste estudo. Diante disso, sugere-se o desenvolvimento de novas pesquisas cuja finalidade seja avaliar as novas formas de cuidados prestados pela equipe de enfermagem aos portadores de hipertensão, considerando a importante magnitude da temática para a Saúde Pública.

\section{Referências}

Amaral-Moreira, B. M., Moura-Lanza, F. \& Nogueira-Cortez, D. (2019). Efetividade da consulta de enfermagem na adesão ao tratamento da hipertensão arterial sistèmica. Rev. Salud Pública, 21(3), 1-9. https://doi.org/10.15446/rsap.v21n3.70291

Brasil. (2019). Secretaria de Vigilância em Saúde. vigilância de fatores de risco e proteção para doenças crônicas por inquérito telefônico. Brasília: Ministério da Saúde

Cavalcante, T. F., Oliveira, L. R., Gondim, A. L. M., Ferreira, J. E., S. M., Nemer, A. P. L., \& Moreira, R. P. (2020). Avaliação da satisfação do resultado de enfermagem Bem-estar Pessoal em idosos com doenças crônicas. Revista Eletrônica De Enfermagem, 22. https://doi.org/10.5216/ree.v22.58690

COFEN. (2009). Conselho Federal de Enfermagem. Resolução COFEN ñ 358/2009, de 15 de outubro de 2009. Sistematização da Assistência de Enfermagem e a implementação do Processo de Enfermagem em ambientes, públicos ou privados, em que ocorre o cuidado profissional de Enfermagem, e dá outras providências.

Chaves, R., Silva, C., Motta, E., Ribeiro, E., \& Andrade, Y. (2016). Sistematização da assistência de enfermagem: visão geral dos enfermeiros. Revista de Enfermagem UFPE on line, 10(4), 1280-1285. https://doi.org/10.5205/1981-8963-v10i4a11114p1280-1285-2016

Daniel, A. C. Q. G., Pedrosa, R. B. S., \& Veiga, E. V. (2018). Cuidados de enfermagem em crise hipertensiva: uma revisão integrativa. Rev. Soc. Cardiol. Estado de Säo Paulo, 28(3).

Herdman, T. H., \& Kamitsuru, S. (2018). Diagnósticos de enfermagem da NANDA: definições e classificação 2018-2020, 11º edição / NANDA International; tradução Regina Machado Garcez. - Porto Alegre: Artmed.

Lima, C. C., Balbino, P. C., Souza, C. C., \& Salgado, P. O. (2017). Diagnósticos de enfermagem identificados em sujeitos portadores de hipertensão arterial. Enfermagem Brasil, 16(5). https://doi.org/10.33233/eb.v16i5.1028

Maia, J. D. S., Silva, A. B., Melo, R. H. V., Rodrigues, M. P., \& Junior, A. M. (2018). A Educação em Saúde para usuários hipertensos: percepções de profissionais da Estratégia Saúde da Família. Revista Ciência Plural, 4(1), 81-97. Recuperado de https://periodicos.ufrn.br/rcp/article/view/13634 
Research, Society and Development, v. 10, n. 12, e219101219269, 2021

(CC BY 4.0) | ISSN 2525-3409 | DOI: http://dx.doi.org/10.33448/rsd-v10i12.19269

Malta D. C., Silva M. A., Moura, L., \& Neto O.L.M. (2017). A implantação do Sistema de Vigilância de Doenças Crônicas Não Transmissíveis no Brasil, 2003 a 2015: alcances e desafios. Rev. bras. Epidemiol, 20 (04), 661-675. https://doi.org/10.1590/1980-5497201700040009.

Mendes, F., Silva, M., \& Ferreira, C. (2018). Diagnósticos de enfermagem em portadores de hipertensão arterial na atenção primária. Estação Científica (UNIFAP), 8(1), 91-101. http://dx.doi.org/10.18468/estcien.2018v8n1.p91-101

Pinto, E. S. O., \& Rodrigues, W. N. (2018). Sistematização da Assistência de Enfermagem na Atenção Primária a pessoas portadoras de hipertensão arterial. Nursing (Säo Paulo), 21(237), 2036-2040.

Reis, L. L. M., Silva, R. C. M., Silva, H. C. D. A., Silva, M. R. B., Souza, C. C., \& Cunha, A. L (2018). Métodos não farmacológicos utilizados pelo Enfermeiro na prevenção e controle da Hipertensão Arterial Sistêmica. Nursing USP, 21(244), 2338-2341. http://portal.fundacaojau.edu.br:807 7/sif/revista_nursing/RevistaNursing_244.pdf

Salles, A., Sampaio, C., Pereira, L., Malheiros, N., \& Gonçalves, R. (2019). O enfermeiro e a questão da adesão do paciente ao tratamento da hipertensão arterial sistêmica. Revista Enfermagem UERJ, 27, e37193.https://doi.org/10.12957/reuerj.2019.37193

Sociedade Brasileira de Cardiologia. (2016). Sociedade Brasileira de Hipertensão, Sociedade Brasileira de Nefrologia. VI Diretrizes Brasileiras de Monitorização Ambulatorial da Pressão Arterial (MAPA) e III Diretrizes Brasileiras de Monitorização Residencial de Pressão Arterial (MRPA). Arq Bras Cardiol.

Sociedade Brasileira de Hipertensão. (2020). Sociedade Brasileira de Nefrologia. VII Diretriz Brasileira de Hipertensão Arterial. Arq Bras Cardiol.

Sociedade Brasileira de Cardiologia. (2017). $7^{\mathrm{a}}$ Diretriz brasileira de hipertensão arterial. Rev Bras Hipertens.

Sousa, L. S., Pessoa, M. S. A., Oliveira, R. P. P., Meneses, A. S. S., Costa, L.M., Alves, N. R., \& Almeida, T.C F. (2019). Caracterização sociodemográfica e clínica dos pacientes hipertensos não controlados atendidos em uma unidade de pronto atendimento), Nursing (Säo Paulo 22(255): 3088-3094.

Sousa, A., Moreira, T., Machado, A., \& Silva, A. (2018). Associação entre adesão ao tratamento anti-hipertensivo e integralidade no atendimento de enfermeiros. Revista Enfermagem UERJ, 26, e25250. https://doi.org/10.12957/reuerj.2018.25250

Vasconcelos, M. I. O., Farias, Q. L. T., Nascimento, F. G., Cavalcante, A. S. P., Mira, Q. L. M., \& Queiroz, M. V. O. (2017). Educação em saúde na atenção básica: uma análise das ações com hipertensos. Rev. APS; 20(2): 253-262. https://doi.org/10.34019/1809-8363.2017.v20.15943

Vieira, V. A. S., Azevedo, C., Sampaio, F. C., Oliveira, P. P., Moraes, J. T., \& Mata, L. R. F. (2017). Cuidados de Enfermagem para pessoas com Diabetes Mellitus e Hipertensão Arterial: mapeamento cruzado. Rev. baiana enferma., Salvador, 31(4). https://doi.org/10.18471/rbe.v31i4.21498 